\title{
Serine/Threonine-Protein Kinase ICK
}

National Cancer Institute

\section{Source}

National Cancer Institute. Serine/Threonine-Protein Kinase ICK. NCI Thesaurus. Code C150263.

Serine/threonine-protein kinase ICK (632 aa, $71 \mathrm{kDa}$ ) is encoded by the human ICK gene. This protein plays a role in serine/threonine-protein kinase activity, ciliogenesis and intraflagellar transport. 